\title{
Fonctionnement des élevages camelins de la zone périurbaine d'Agadez au Niger : enquête typologique
}

\author{
M. Chaibou ${ }^{1}$ B. Faye ${ }^{2 *}$
}

\begin{abstract}
Mots-clés
Camelus dromedarius - Conduite d'élevage - Classification - Zone périurbaine - Agadez - Niger.
\end{abstract}

\begin{abstract}
Résumé
Le cheptel camelin est tributaire des ressources fourragères des parcours naturels dont le développement est soumis aux aléas de la pluviométrie, très mal repartie dans le temps et l'espace. Pour exploiter ces parcours, I'une des stratégies adoptée par les éleveurs est la mobilité. Cependant, depuis un certain temps, les mutations climatiques, socio-économiques et les conditions d'une urbanisation accélérée imposent de plus en plus des changements dans la règle de gestion des élevages. Le développement à Agadez d'une minilaiterie, dont l'activité principale est centrée essentiellement sur la transformation du lait de chamelle, nécessite la connaissance parfaite du fonctionnement des élevages laitiers qui vont approvisionner cette laiterie. Une enquête a été menée durant deux mois auprès de cent élevages camelins de la zone périurbaine d'Agadez en vue de connaître le fonctionnement des élevages de cette zone. L'analyse des données recueillies sur les pratiques de gestion de troupeaux et le fonctionnement global des élevages des pasteurs chameliers ont permis de distinguer trois grands types d'élevages, différenciés essentiellement sur la base des pratiques d'alimentation des animaux et sur la mobilité des pasteurs. A cela s'est ajoutée une affinité zonale de certains éleveurs, dont les causes sont sociohistoriques et écologiques. Le premier groupe de pasteurs avait la particularité d'être sédentaire, d'avoir un troupeau de taille moyenne et de pratiquer la complémentation et la spéculation laitière. Le deuxième groupe pratiquait fortement la transhumance en saison des pluies et certains d'entre eux complémentaient les animaux. La vente du lait de chamelle était quasi inexistante pour ces pasteurs. Les chameliers composant le troisième groupe avaient de grands troupeaux et pratiquaient en conséquence un nomadisme régulier. Par les contacts particuliers établis par certains producteurs de ce groupe avec la laiterie, la vente de lait était de plus en plus pratiquée. La taille, la composition des troupeaux, les ressources fourragères et hydrauliques constituaient les principaux facteurs déterminants des déplacements des éleveurs.
\end{abstract}

\section{INTRODUCTION}

Les systèmes d'élevage camelins des zones arides et subarides d'Afrique noire sont assez peu connus, contrairement à ceux d'Afrique du Nord (13). L'élevage camelin, jadis exclusivement extensif, se trouve aujourd'hui en passe à des changements eu égard aux mutations écologiques dans les milieux de production, aux mutations socio-économiques intervenues ces dernières années dans les

1. Université Abdou Moumouni, Niamey, Niger.

2.Cirad, Emvt, Direction, Montpellier, F-34000 France.

* Auteur pour la correspondance

Cirad, TA 30/A, Campus international de Baillarguet,

34398 Montpellier Cedex 5, France.

Tél. : + 33 (0)4 67593703 ; fax : +33 (0)4 67593825

E-mail : faye@cirad.fr sociétés pastorales, mais aussi à un accroissement démographique marqué des centres urbains $(4,12)$. Ce grossissement des villes a de fait augmenté les besoins alimentaires et en particulier en lait des populations urbaines. C'est le cas du lait de chamelle, dont la demande s'est accrue particulièrement dans les provinces sahariennes (Agadez, Tabalak) où l'on trouve généralement un important élevage camelin (6). Le lait de chamelle contribue ainsi de plus en plus à l'alimentation des populations urbaines de certaines villes africaines, alors qu'il a longtemps été destiné à l'autoconsommation (5).

On peut citer à ce titre l'exemple de la ville d'Agadez, où un élevage camelin périurbain est en train de se développer à la faveur de la mise en place d'une unité de transformation laitière (4). Dans ces conditions, les éleveurs camelins sont de plus en plus nombreux à venir s'installer aux environs de la ville, certains définitivement, d'autres de manière saisonnière, pour bénéficier d'un meilleur 
accès aux intrants ou aux marchés. Ces élevages camelins assurent l'approvisionnement d'une unité de transformation de lait installée dans cette ville d'Agadez. Cependant, on constate d'importantes variations dans les quantités livrées à cette unité de transformation qui semblent liées au mode de fonctionnement de ces élevages.

Partant de l'hypothèse qu'il existait une dynamique dans l'ensemble de la zone entraînant d'importants changements (en particulier socio-économiques) au niveau de la ville, une étude typologique du système a été réalisée pour appréhender le fonctionnement des élevages $(9,11)$ et leurs contraintes afin de mieux orienter des opérations de développement. Ce travail, basé sur une enquête typologique des élevages localisés dans cette zone périurbaine d'Agadez, vise à connaître les types d'élevages selon leur mode de fonctionnement (16). L'approche est basée essentiellement sur les méthodes typologiques (17). L'étude a concerné principalement la mobilité des pasteurs et leurs troupeaux ainsi que le système d'alimentation et de gestion des troupeaux. Elle a été menée du 6 septembre au 25 octobre 2002.

\section{MATERIEL ET METHODES}

\section{Milieu physique et humain}

\section{Climat}

Le cadre de l'étude est constitué par la zone périurbaine d'Agadez, située à $910 \mathrm{~km}$ au nord-est de Niamey (figure 1). Autour de la ville d'Agadez plusieurs paysages sont présents. C'est le cas du massif montagneux de l'Aïr, vaste plateau compris entre 500 et $2500 \mathrm{~m}$ d'altitude où alternent des regs (déserts caillouteux), des affleurements, des vallées, des plaines alluviales et des reliefs volcaniques.

Le sud-ouest d'Agadez est formé par des plaines d'inondation dont les principales sont les plaines de l'Azawak et de l'Irazher, alimentées par un réseau de koris orientés généralement d'est en ouest (de zones de plateaux aux vallées). L'étude a donc été menée dans une zone désertique, où la pluviométrie annuelle moyenne est inférieure à $150 \mathrm{~mm}$. Le climat est de type aride (pluviométrie inférieure à $150 \mathrm{~mm}$ par an) et de transition saharo-sahélienne. En 2003 les précipitations enregistrées à la station météorologique d'Agadez étaient de $142 \mathrm{~mm}$. La variation interannuelle est très importante (4,2 à 288,2 mm par an, sur 4 à 44 jours de pluies par an) et suit un gradient dégressif du sud au nord. Ce climat est caractérisé par de grands écarts thermiques nycthéméraux et saisonniers.

\section{Sols et végétation}

Les sols rencontrés sont des sols minéraux bruts, avec notamment les lithosols, constitués par l'ensemble des débris grossiers et fins qui s'accumulent au pied des versants, et les régosols qui sont à l'origine des regs et des sols d'apports fluviatiles.

La végétation de cette zone est très pauvre : c'est une steppe très clairsemée. Les principales espèces rencontrées sont Acacia sp. (Acacia raddiana, Acacia nilotica, Acacia erhenbergiana) Boscia senegalensis, Maerua crassifolia, Salvadora persica et Calotropis

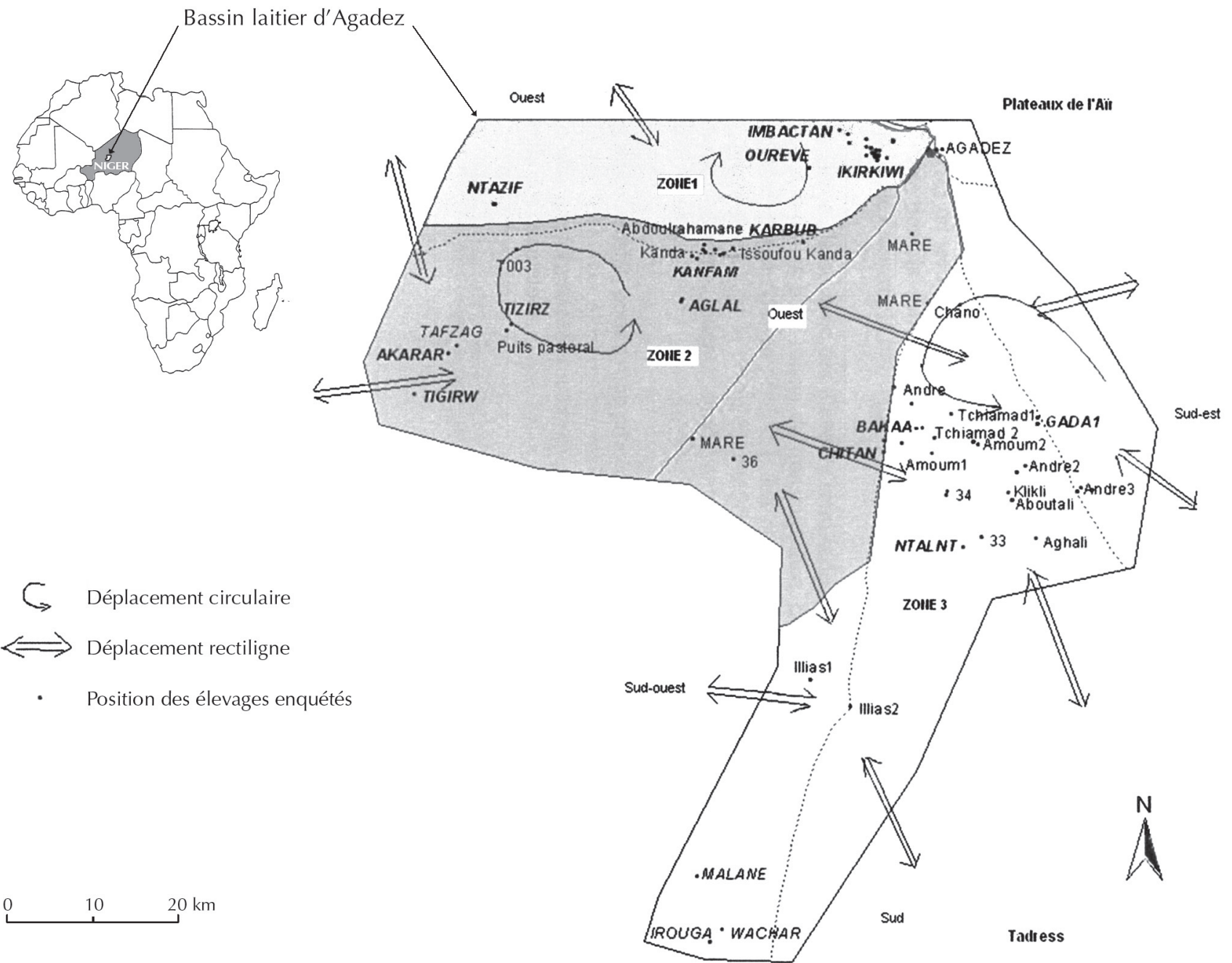

Figure 1 : déplacements des éleveurs et situation géographique de la zone d'étude. 
procera. Le tapis herbacé est essentiellement graminéen. On trouve des espèces comme Eragrostis tremula, Chloris pilosa, Cenchrus biflorus, Lasiurus hirsutus, Panicum turgidum, Aristida adscensionis. D'autres espèces d'importance fourragère ont également été décrites : il s'agit de Schouwia thebaica, Tribulus longipetalus et Caralluma russeliana.

\section{Peuplement urbain et rural}

La ville d'Agadez compte, en 2003, 162000 habitants selon la direction des statistiques nationales. Cette population est composée essentiellement de Touaregs (kel Aïr), de Haoussas, de Djermas, d'Arabes et, dans une moindre mesure, de Peulhs et de Kanouris. En dehors des Arabes et des Peulhs, le milieu rural est principalement peuplé par les Touaregs. Ce groupe est divisé en plusieurs sous-groupes (une quinzaine). Cependant, dans la zone d'étude, on trouve surtout les Touaregs kel Ferwan, les kel Ewey, les kel Gharus et les kel Tadele (8).

\section{Systèmes d'élevage de la zone}

Le système d'élevage qui prédomine est le système d'élevage extensif pastoral. Ce système est basé essentiellement sur la mobilité des troupeaux. En revanche, deux modes de conduite d'élevage existent : éleveurs installés en zone périurbaine qui pratiquent la transhumance de saison des pluies et éleveurs qui se déplacent sans cesse sous forme de nomadisme.

Cependant un système semi-intensif se met en place, essentiellement localisé en zone périurbaine d'Agadez. Dans ce système, les éleveurs utilisent des intrants zootechniques en complémentation. La zone périurbaine d'activité pastorale occupe la partie ouest, le sud-ouest et le sud de la ville. Elle correspond à la commune urbaine d'Agadez et couvre une superficie d'environ $4424 \mathrm{~km}^{2}$. Suite à plusieurs missions de reconnaissance, les auteurs ont subdivisé la zone périurbaine en trois sous-zones identifiées selon leurs caractéristiques écologiques (figure 1).

- Sous-zone 1 (zone ouest)

C'est la zone de ruissellement du Kori Teloua. D'autres réseaux de ruissellement des pentes voisines confluent et forment des mares temporaires. Les sols sont argileux compacts au niveau des dépressions, et sablonneux par endroits du fait de l'accumulation d'éléments fins venus des zones d'altitude.

Cette zone est comprise entre la route de Tahoua à Agadez et la route d'In'Gall. La végétation de type steppique est très clairsemée et constituée essentiellement d'arbustes, essentiellement des Acacia (raddiana, ehrenbergiana, nilotica...) et d'autres plantes, surtout Salvadora persica spécifique à cette zone, mais aussi Balanites aegyptiaca, Boscia senegalensis, Maerua crassifolia et Calotropis procera; le tapis herbacé est majoritairement constitué de graminées. C'est une zone de passage importante pour les éleveurs de bovins peulhs venant des zones agropastorales du sud de Tahoua et Zinder, et transhumant vers la zone nord-ouest d'Azag, réputée pour ses herbages riches en sels. Dans cette zone périurbaine, les éleveurs camelins se sont fixés. Certains d'entre eux ont construit des habitations plus pérennes en banco et ne se déplacent guère. La très grande proximité de la ville $(7 \mathrm{~km})$ leur permet de bénéficier de nombreux avantages, notamment l'accès rapide aux intrants mais également au débouché laitier.

\section{- Sous-zone 2 (zone sud-ouest)}

Elle est constituée par une vaste plaine inondable de part et d'autre de la route d'Agadez à Tahoua. C'est la grande plaine de l'Irhazer qui représente le plus vaste parcours potentiellement exploité par les pasteurs chameliers de la zone et/ou transhumants. Le substrat de cette zone est constitué d'éléments sablo-rocailleux et d'un sol argileux dans les parties dépressionnaires (19). Excepté la steppe arbustive de Kerboubou, la strate arbustive est quasi inexistante.

- Sous-zone 3 (zone sud)

Cette zone s'étendant jusqu'aux falaises de Tiguidit est traversée essentiellement par trois grandes vallées fossiles (Tassack'n talam, Adrar zaggren, et Tchiffaye n'jen) avec de nombreuses surfaces occasionnellement humides, des lits d'oueds, de grandes étendues de sables latéritiques et de nombreux affleurements caillouteux. C'est la zone localisée de part et d'autre de la route d'Agadez à Zinder. La strate arbustive, en dehors des alentours de la mare de Tassack'n talam, est quasi inexistante. Quelques pousses de Boscia senegalensis et de Maerua crassifolia sont éventuellement rencontrées. Au-delà des falaises de Tiguidit se trouvent de grands élevages détenus par des Arabes et autres Touaregs kel Ferwan, exploitant les vastes parcours de Tadress qui s'étendent jusqu'à Aderbissinat.

\section{Méthodologie d'enquête}

Les auteurs se sont intéressés aux différentes pratiques de gestion des élevages par les pasteurs qui leur permettent de gérer et de conduire leur cheptel. Un questionnaire a été établi sur la base de ces différents aspects. Deux grands thèmes ont été visés : les pratiques portant sur le troupeau et les pratiques portant sur l'utilisation des parcours et de l'espace en général.

Concernant la gestion du troupeau, les points suivants ont été mis en exergue :

- la constitution du troupeau ;

- le mode d'alimentation des animaux ;

- la valorisation des productions ;

- la pratique de la traite et la lactation;

- la gestion de la reproduction.

Le deuxième thème a concerné l'étude du mode d'exploitation des pâturages et des stratégies de déplacement.

Cette enquête a été menée auprès des élevages camelins accessibles de la zone d'étude. L'unité d'enquête a donc été le troupeau. Cette enquête a été basée sur des entretiens menés avec les chefs de famille responsables des élevages. Quinze campements ont été visités pour conduire ces travaux, soit cinq par sous-zone (tableau I). L'ensemble des résultats obtenus a permis non seulement de construire une typologie des élevages périurbains, mais aussi de caractériser les principales pratiques de gestion de troupeaux.

Les élevages enquêtés représentaient 13 p. 100 des élevages présents dans la zone périurbaine. Le questionnaire d'enquête,

\section{Tableau I}

Campements concernés par l'enquête

\begin{tabular}{lll}
$\begin{array}{l}\text { Zone ouest } \\
\text { (zone 1) }\end{array}$ & $\begin{array}{l}\text { Zone sud-ouest } \\
\text { (zone 2) }\end{array}$ & $\begin{array}{l}\text { Zone sud } \\
\text { (zone 3) }\end{array}$ \\
\hline Ikirkiwi 1 & Kerboubou 1 & Tassack'n talam \\
Ikirkiwi 2 & Kerboubou 2 & Gada \\
Dari & Kanfamata & Chitan \\
Imbackatane & Akarara & Abalama \\
Oureille & Aglal'n gar & Wacharou
\end{tabular}


construit sur la base des principaux thèmes cités ci-dessus, a abordé tous les sujets touchant au troupeau. Le questionnaire a été élaboré sous forme de fiches, l'unité de base retenue a été le troupeau, défini comme l'unité de gestion des animaux au moment de l'enquête. Deux enquêteurs ont été formés pour conduire ce travail sur le terrain. Le questionnaire a été testé dans un premier temps au cours d'une pré-enquête, avant d'être utilisé au niveau de tous les campements. Cent élevages ont été enquêtés durant environ deux mois. Les données obtenues ont été saisies sur les logiciels de bases de données Access et Excel. Les auteurs ont procédé au codage des variables, à la définition et au codage des modalités, à la suppression des variables redondantes ou inexploitables (13). Pour l'analyse des données ont été utilisés les logiciels XLstat et R. L'analyse a été faite dans un premier temps sur les différents thèmes, pour identifier le thème le plus discriminant sur les élevages. A l'issue de cette phase, deux variables de gestion des troupeaux (le mode d'alimentation et le déplacement) ont été retenues pour construire la typologie des élevages. Une analyse factorielle des correspondances multiples (Afcm), puis une classification ascendante hiérarchique $(\mathrm{CAH})$ sur les facteurs obtenus ont permis de construire une typologie synthétique des élevages de cette zone périurbaine d'Agadez.

\section{RESULTATS}

Tous les troupeaux étaient détenus par des pasteurs touaregs. Les troupeaux comprenaient toujours des dromadaires généralement associés à des caprins, des ovins, et des asins. Dans de rares cas, des bovins étaient présents dans le troupeau. Parmi les éleveurs enquêtés, 38 p. 100 étaient localisés dans la zone 3 (zone sud), 28 p. 100 dans la zone 1 (zone ouest) et 34 p. 100 dans la zone 2 (zone sud-ouest). Les dromadaires ont représenté l'espèce la plus importante du point de vue numérique. Un troupeau individuel comptait en moyenne $24 \pm 11$ dromadaires dans la zone 1, $22 \pm 1$ dromadaires dans la zone 2 et $35 \pm 10$ dromadaires dans la zone 3 . Quant aux petits ruminants, il y avait en moyenne $13 \pm 6$ ovins et $21 \pm 9$ caprins par troupeau individuel sur l'ensemble de la zone périurbaine d'Agadez.

\section{Analyse typologique des données}

Une typologie des élevages a été réalisée à l'aide d'une Afcm et d'une CAH. Plusieurs variables ont été prises en compte dans cette typologie (tableau II).

\section{Tableau II}

Modalités des variables utilisées dans la typologie (100 troupeaux enquêtés) et leurs contributions aux facteurs principaux

\begin{tabular}{|c|c|c|c|c|c|c|}
\hline Code & Variables actives & Modalité & de troupeaux & $\mathrm{F} 1(\%)$ & F2 (\%) & F3 $(\%)$ \\
\hline \multirow[t]{4}{*}{ ModElev } & Mode d'élevage & 1. Semi-sédentaire (Ss) & 34 & 0,0 & 14,3 & 0,1 \\
\hline & & 2. Micronomadisme (Mn) & 38 & 8,4 & 4,5 & 0,0 \\
\hline & & 3. Sédentaire $(\mathrm{S})$ & 28 & 10,9 & 2,9 & 0,0 \\
\hline & & Total ModElev & & 19,3 & 21,7 & 0,1 \\
\hline \multirow[t]{8}{*}{ RaModEl } & Raison de ce mode d'élevage & 1. Recherche d'eau (RE) & 2 & 0,1 & 2,2 & 0,2 \\
\hline & & 2. Recherche d'eau + pâturage (RE + RP) & 36 & 5,3 & 2,0 & 0,3 \\
\hline & & $\begin{array}{l}\text { 3. Recherche d'eau + pâturage + sel } \\
(\mathrm{RE}+\mathrm{RP}+\mathrm{RS})\end{array}$ & 16 & 1,1 & 2,1 & 1,0 \\
\hline & & 4. Recherche de pâturage (RP) & 3 & 0,1 & 0,9 & 6,0 \\
\hline & & 5. Terroir d'attache $(\mathrm{T})$ & 33 & 5,2 & 0,1 & 2,4 \\
\hline & & 6. Terroir d'attache + vente de lait $(T+V L)$ & 3 & 1,0 & 0,0 & 0,7 \\
\hline & & 7. Vente de lait $(\mathrm{VL})$ & 7 & 2,3 & 0,9 & 1,4 \\
\hline & & Total RaModElev & & 15,1 & 8,3 & 12,1 \\
\hline \multirow[t]{6}{*}{ FreqDplcmt } & Fréquence des déplacements & 1. Chaque deux à trois mois (2 à 3 mois) & 2 & 0,1 & 1,6 & 7,5 \\
\hline & & 2. Chaque six mois (6 mois) & 3 & 0,0 & 2,2 & 2,1 \\
\hline & & 3. Aucun déplacement (aucun dplct) & 26 & 11,3 & 3,9 & 0,0 \\
\hline & & 4. Chaque année (chaq an) & 27 & 0,0 & 11,3 & 1,9 \\
\hline & & 5. Indéterminée (indifini) & 42 & 7,6 & 3,3 & 0,0 \\
\hline & & Total FreqDplcmt & & 19,1 & 22,3 & 11,5 \\
\hline \multirow[t]{7}{*}{ OrDplcmt } & Direction habituelle (sens) & 1. Est (Est) & 4 & 0,0 & 2,6 & 10,1 \\
\hline & prise lors des déplacements & 2. Ouest (Ou) & 19 & 0,0 & 11,2 & 4,1 \\
\hline & & 3. Pas de déplacement (Pdpl) & 28 & 11,2 & 3,2 & 0,0 \\
\hline & & 4. Sud-est (SE) & 9 & 1,5 & 0,1 & 0,5 \\
\hline & & 5. Sud-ouest (SO) & 33 & 5,6 & 2,4 & 0,3 \\
\hline & & 6. Sud (sud) & 7 & 0,4 & 0,3 & 2,1 \\
\hline & & Total OrDplcmt & & 18,8 & 19,8 & 17,0 \\
\hline
\end{tabular}


Tableau II (suite)

\begin{tabular}{|c|c|c|c|c|c|c|}
\hline Code & Variables actives & Modalité & de troupeaux & F1 (\%) & F2 (\%) & F3 (\%) \\
\hline \multirow[t]{7}{*}{ Nbritin } & \multirow{7}{*}{$\begin{array}{l}\text { Nombre d'itinéraires choisis lors } \\
\text { de déplacement avant destination }\end{array}$} & 1. Aucun itinéraire (aucun) & 46 & 5,0 & 0,8 & 0,3 \\
\hline & & 2. Cinq itinéraires (cinq) & 1 & 0,0 & 0,2 & 0,7 \\
\hline & & 3. Dix itinéraires (dix) & 1 & 0,4 & 0,8 & 1,3 \\
\hline & & 4. Indéterminé (indfini) & 8 & 2,2 & 1,6 & 0,8 \\
\hline & & 5. Deux itinéraires (deux) & 37 & 3,0 & 0,9 & 2,8 \\
\hline & & 6. Trois itinéraires (trois) & 7 & 0,0 & 2,9 & 12,6 \\
\hline & & Total Nbritin & & 10,5 & 7,3 & 18,5 \\
\hline \multirow[t]{8}{*}{ PsaiCompl } & \multirow{8}{*}{$\begin{array}{l}\text { Pratique et saison } \\
\text { de complémentation }\end{array}$} & 1. Aucune complémentation (ac) & 52 & 4,5 & 0,0 & 0,3 \\
\hline & & 2. Saison pluvieuse (sp) & 1 & 0,0 & 0,1 & 1,2 \\
\hline & & $\begin{array}{l}\text { 3. Saison pluvieuse + saison sèche froide } \\
(\mathrm{Sp}+\mathrm{ssf})\end{array}$ & 2 & 0,3 & 0,2 & 0,3 \\
\hline & & 4. Saison sèche chaude (ssc) & 28 & 4,9 & 0,3 & 1,4 \\
\hline & & $\begin{array}{l}\text { 5. Saison sèche chaude + saison pluv. } \\
(\mathrm{ssc}+\mathrm{sp})\end{array}$ & 1 & 0,0 & 1,6 & 11,7 \\
\hline & & $\begin{array}{l}\text { 6. Saison froide }+ \text { saison sèche chaude } \\
(\mathrm{ssf}+\mathrm{ssc})\end{array}$ & 13 & 0,6 & 0,0 & 0,0 \\
\hline & & 7. Toute saison (ts) & 3 & 0,9 & 0,0 & 10,6 \\
\hline & & Total PsaiCompl & & 11,2 & 2,3 & 25,4 \\
\hline \multirow[t]{3}{*}{ PratqTrans } & \multirow{3}{*}{$\begin{array}{l}\text { Pratique de transhumance en } \\
\text { saison des pluies }\end{array}$} & 1. Oui (Otrans) & 59 & 2,0 & 4,9 & 0,0 \\
\hline & & 2. Non (trans) & 41 & 1,4 & 3,4 & 0,0 \\
\hline & & Total PratqTrans & & 3,3 & 8,4 & 0,0 \\
\hline \multirow[t]{5}{*}{ NbCAM } & \multirow{5}{*}{$\begin{array}{l}\text { Effectif de dromadaires } \\
\text { dans le troupeau }\end{array}$} & 1. Moins de 20 têtes(cam1) & 34 & 0,3 & 1,5 & 0,1 \\
\hline & & 2. 21 à 50 têtes (cam2) & 58 & 0,1 & 0,2 & 1,8 \\
\hline & & 3. 51 à 100 têtes (cam3) & 7 & 0,1 & 1,0 & 7,6 \\
\hline & & 4. Plus de 100 têtes (cam4) & 1 & 0,5 & 0,5 & 0,7 \\
\hline & & Total NbCAM & & 1,0 & 3,3 & 10,1 \\
\hline \multirow[t]{7}{*}{ Propautoc } & \multirow{6}{*}{$\begin{array}{l}\text { Proportion de lait produit } \\
\text { autoconsommé }\end{array}$} & 1. $30 \%$ autoconsommé & 4 & 0,5 & 2,6 & 0,6 \\
\hline & & 2. $50 \%$ autoconsommé & 24 & 1,1 & 1,2 & 0,5 \\
\hline & & 3. $70 \%$ autoconsommé & 31 & 0,0 & 3,0 & 1,4 \\
\hline & & 4. $100 \%$ autoconsommé & 41 & 0,1 & 0,0 & 2,8 \\
\hline & & Total Propautoc & & 1,7 & 6,8 & 5,3 \\
\hline & & & & 100 & 100 & 100 \\
\hline & \multicolumn{3}{|l|}{ Variables supplémentaires } & & & \\
\hline \multirow[t]{3}{*}{ Localis } & \multirow{3}{*}{$\begin{array}{l}\text { Localisation de l'élevage } \\
\text { enquêté par rapport à Agadez }\end{array}$} & 1. Ouest (zone 1) & 28 & & & \\
\hline & & 2. Sud-ouest (zone 2) & 34 & & & \\
\hline & & 3. Sud (zone 3) & 38 & & & \\
\hline \multirow[t]{4}{*}{ EffTotal } & \multirow{4}{*}{$\begin{array}{l}\text { Effectif total d'animaux dans } \\
\text { le troupeau }\end{array}$} & 1. Moins de 50 têtes (effetot1) & 30 & & & \\
\hline & & 2. 50 à 100 têtes (effetot2) & 58 & & & \\
\hline & & 3. 101 à 150 têtes (effetot3) & 11 & & & \\
\hline & & 4. 151 à 200 têtes (effetot4) & 1 & & & \\
\hline
\end{tabular}

L'analyse de l'histogramme des valeurs propres issu de l'Afcm a permis d'identifier le nombre d'axes à considérer. L'ensemble de trois axes a expliqué globalement 29,2 p. 100 d'inertie (figures 1 et 2). L'analyse des contributions a permis de juger si une variable était bien représentée sur un axe, permettant ainsi d'identifier celles qui expliquaient les principaux axes. Les principales variables qui présentaient des contributions fortes étaient le mode d'élevage (ModElev) avec 19,3 p. 100, la fréquence de déplacement (FréqDplcmt) avec 19,05 p. 100, la direction habituelle prise lors des déplacements (OrDplcmt) dont la contribution était de 18,8 p. 100 et la raison du mode d'élevage 
(RaModElev) avec 15,1 p. 100 . Toutes ces variables expliquaient l'axe F1. L'axe F2 était également mieux représenté par des variables qui étaient en relation avec les déplacements, notamment la fréquence de déplacement pour 22,3 p. 100, le mode d'élevage avec 21,7 p. 100 , l'orientation des déplacements pour 19,8 p. 100 et la pratique de transhumance (PratqTrans) avec une contribution de 8,4 p. 100.

La variable déplacement a contribué fortement à l'explication des axes et donc à la distinction des groupes. Mais le mode de conduite d'élevage ainsi que la complémentation ont également contribué à distinguer les élevages. Cependant, l'influence des facteurs F2 et F3 étaient d'un intérêt secondaire, permettant de retenir l'axe F1 comme l'axe principal qui expliquait la partie la plus importante de l'information. Globalement, les facteurs F1 et F2 ont permis de mieux expliquer la situation des individus selon les variables considérées. L'échelle des contributions des variables aux axes factoriels est représentée par le tableau II.

La variable déplacement a pris une place importante dans l'analyse. En effet, les éleveurs se sont différenciés essentiellement par leur mode de déplacement, les raisons des déplacements, le mode d'alimentation des animaux, leurs zones de localisation et les effectifs de leurs troupeaux.

\section{Identification des groupes d'élevages laitiers camelins}

L'examen du plan factoriel des modalités (figure 2) a permis de visualiser dans un premier temps les différentes corrélations possibles entre les variables. L'axe F1 se rapportait à deux groupes d'élevages qui s'opposaient, avec un premier groupe (côté négatif) dont les caractéristiques liées entre elles étaient les suivantes :

- ils ne pratiquaient aucune complémentation (ac), le mode d'élevage était le micronomadisme (Mn), ils avaient un nombre indéterminé d'itinéraires lors des déplacements au cours de l'année ;

- ils avaient des effectifs assez élevés de dromadaires (de 51 à 100) et d'une manière générale un cheptel important (effetot4) avec plus de 150 têtes et ne pratiquaient pas la transhumance (0trans), leurs déplacements étaient essentiellement motivés par la recherche des points d'eau et des pâturages (RE + RP).

Le deuxième groupe d'élevages (côté positif) présentait les caractéristiques suivantes :

- ils ne se déplaçaient pas ou le faisaient sur des distances négligeables (Aucundplct) et pratiquaient occasionnellement la transhumance ;

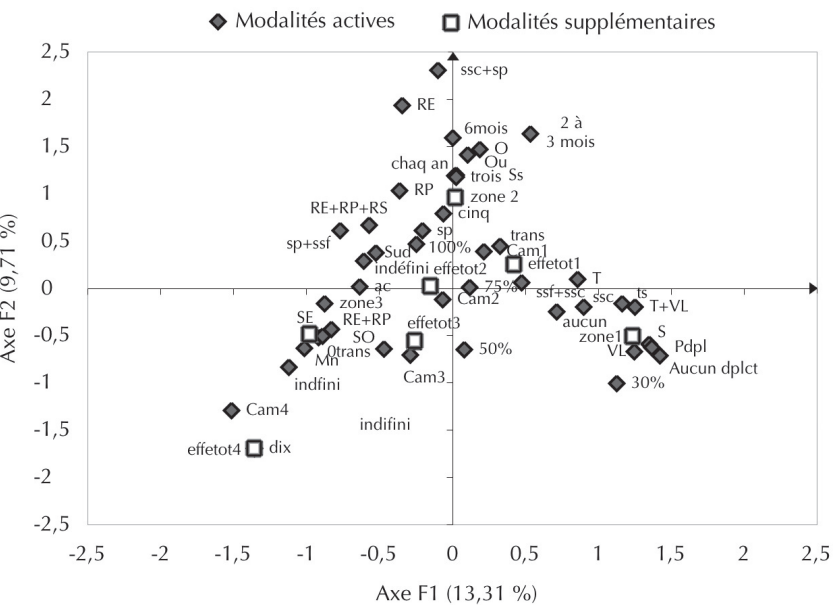

Figure 2: analyse factorielle de correspondance multiple, graphe des modalités.
- ils avaient adopté cependant un mode sédentaire (S) dont la raison principale était que cette zone constituait leur zone d'attache du fait des points d'eau qu'ils avaient eux-mêmes créés ; une deuxième raison avancée était la vente du lait produit par leurs troupeaux $(\mathrm{T}+\mathrm{VL}$ et VL) ;

- certains pratiquaient la complémentation en toute saison, soit au sel, aux graines de coton ou aux fanes de niébé.

L'axe F2 (qui présentait la distribution des situations extrêmes) décrit un troisième groupe dont les caractéristiques étaient les suivantes :

- en haut de l'axe, se trouvaient des éleveurs qui se déplaçaient chaque six mois pour certains et chaque deux à trois mois pour d'autres dans la même zone (excepté lors de la transhumance), et dont le déplacement était essentiellement motivé par la recherche des points d'eau; leur mode d'élevage était semi-sédentaire, c'est-à-dire qu'ils pratiquaient la transhumance $(\mathrm{Ss})$; ils pratiquaient également la complémentation en saison sèche et en saison pluvieuse $(\mathrm{ssc}+\mathrm{sp})$; ils avaient des effectifs de dromadaires très réduits (cam1, moins de 20 têtes) et d'une manière générale un effectif total faible (effetot1, moins de 50 têtes) ; ils autoconsommaient, pour la majorité, la totalité $(100$ p. 100) de leur production de lait et étaient localisés dans la zone 2 ; en général, ils se déplaçaient vers l'ouest et leur mouvement était motivé par la recherche soit des points d'eau (RE), soit des bons pâturages et des terres salées (RE, RP), soit d'une combinaison RP + RE + RS ;

- à l'opposé (figure 2), se trouvaient les éleveurs qui avaient des effectifs assez importants de dromadaires (Cam3) et par conséquent un cheptel total moyen (effetot3). Ces éleveurs vendaient jusqu'à 50 p. 100 de leur production de lait et ne pratiquaient que rarement la transhumance. Le deuxième groupe était aussi proche de l'axe F1 que de l'axe F2 (figure 2). Le plan factoriel F1-F2 a donc donné la meilleure représentation avec 23,02 p. 100 d'informations expliquées, alors qu'elles ne l'ont été qu'à 19,5 p. 100 avec le plan F1F3 qui n'a pas permis une représentation claire des groupes.

L'analyse factorielle des correspondances multiples réalisée sur les individus et l'examen du meilleur plan factoriel (F1-F2) a donné une représentation très caractéristique et claire de la répartition des individus et a permis ainsi d'identifier des groupes ayant pris les mêmes valeurs pour les mêmes variables (figure 3).

\section{Construction des classes de typologie selon le fonctionnement des élevages}

Les individus ainsi représentés ont pu être classés et hiérarchisés selon leurs activités et le mode de gestion de leurs troupeaux afin d'avoir une meilleure partition. Une classification ascendante

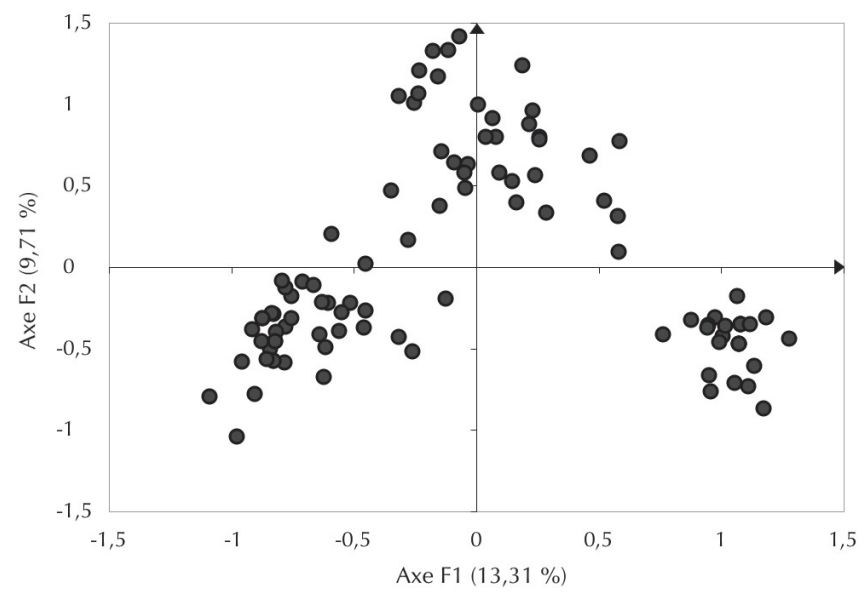

Figure 3 : projections des individus sur le plan F1-F2. 
hiérarchique a conduit à regrouper et à ranger tous les individus en classes en fonction de la « distance » qui les séparait. Cette classification a été réalisée sur la base du nombre d'axes jugés importants en termes d'explication d'inertie sur l'Afcm: les trois premiers axes ont dont été utilisés. La réalisation d'une $\mathrm{CAH}$ à partir des coordonnées de ces axes (F1, F2, F3) et le diagramme des indices des niveaux de regroupement issu de cette analyse ont orienté les auteurs sur le nombre de classes de partition.

C'est donc à partir des grands traits caractéristiques dégagés par l'analyse factorielle que ces partitions ont été construites, ce qui s'inscrivait bien dans la démarche générale d'analyse typologique dont l'objectif final était de construire des ensembles homogènes facilement identifiables. Trois classes de partition ont été obtenues. Le résultat d'une CAH est un arbre hiérarchique appelé aussi dendrogramme qui visualise les différentes partitions successives des individus afin de choisir celles qui paraissent optimales (figure 4). Les trois classes ont été décrites de la façon suivante :

- la classe 1 était constituée par des éleveurs qui pratiquaient la transhumance; leur effectif de dromadaires était faible (moins de 20 têtes) ; ils pratiquaient la complémentation en saison sèche chaude et en sèche froide ; ils se déplaçaient à l'intérieur de la zone 2 chaque six mois pour certains et tous les deux à trois mois pour d'autres ; ils autoconsommaient la totalité de leur production laitière ;

- la classe 2 était formée d'éleveurs sédentarisés situés dans la zone 1 très proche de la ville dont les premiers campements étaient à $7 \mathrm{~km}$ d'Agadez; leurs troupeaux de dromadaires était de taille moyenne (21 à 50 têtes); ils ne pratiquaient aucun déplacement ; ils complémentaient en général en toute saison et vendaient du lait de chamelle dans le centre urbain proche ; dans cette zone, 4 p. 100 des éleveurs vendaient jusqu'à 70 p. 100 de leur production de lait et seulement 3 p. 100 ne vendaient pas leur lait ;

- la classe 3 comportait 38 p. 100 d'éleveurs pratiquant le micronomadisme, tous localisés dans cette zone 3 ; ces éleveurs avaient des effectifs de dromadaires importants (de 50 à 100, parfois plus de 100 têtes) ; ils se déplaçaient selon des itinéraires indéterminés, sur plusieurs directions (sud, sud-est, et ouest); ils vendaient du lait, jusqu'à 50 p. 100 de leur production pour ceux qui avaient un contrat de vente avec la laiterie.

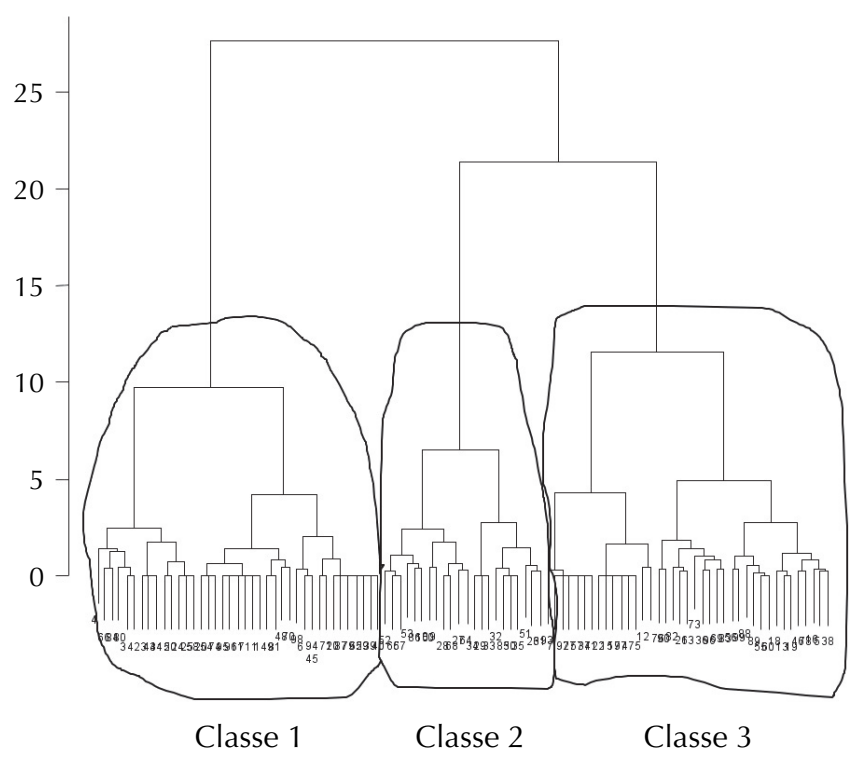

Distance résiduelle

Figure 4 : dendrogramme issu de la classification ascendante hiérarchique des éleveurs.

\section{Pratiques de gestion des troupeaux}

\section{Alimentation des troupeaux}

L'alimentation était basée essentiellement sur le pâturage naturel. Cependant un nombre non négligeable d'éleveurs (48 p. 100) pratiquait la complémentation. La conduite des animaux au pâturage était relativement différente selon l'espèce.

Le troupeau des dromadaires était conduit au pâturage au petit matin. Pour abreuver les animaux, l'éleveur rejoignait le point d'eau avant l'arrivée du troupeau. Une fois abreuvé, le troupeau retournait au pâturage jusqu'au soir. Le temps de pâturage variait en fonction des saisons. Parfois le troupeau passait la nuit au pâturage (situation très fréquente en saison sèche chaude et en période de saillie). Au matin le chamelier vérifiait obligatoirement la localisation de son troupeau. Les chamelles gravides et ayant sevré leurs petits (en général à partir du quatrième mois de gravidité de la femelle) étaient en général allotées. Leur suivi était donc plus aisé que pour les autres. L'éleveur ne vérifiait leur position qu'une fois tous les trois à quatre jours et les abreuvait ou les complémentait à cette occasion.

La surveillance du troupeau de dromadaires était bien moins importante que pour les petits ruminants. La surveillance se limitait à une vérification de la position de troupeau qui se faisait en général une à deux fois par jour, sauf dans des cas particuliers de périodes de recrudescence de vols. Dans tous les autres cas, le troupeau partait sur le parcours le matin et regagnait le parc le soir sans aucun suivi de l'éleveur. Toutefois 45 p. 100 des éleveurs interrogés surveillaient de façon rapprochée leurs troupeaux de dromadaires eux-mêmes ou par l'intermédiaire de leurs enfants. La gestion de l'alimentation faisait intervenir des déplacements saisonniers (transhumance), notamment pendant la saison des pluies. Cette transhumance était motivée en général par la recherche des bons pâturages, mais aussi par la cure salée des animaux.

Cette cure était la période où les transhumants amenaient leurs animaux soit sur des pâturages à herbages riches en sel, soit sur des espaces salés où les animaux léchaient en permanence la terre. Dans tous les cas 46 p. 100 des éleveurs pratiquaient cette transhumance vers la zone d'In' Gall, réputée pour ses terres salées et ses bons pâturages. Certains éleveurs répartissaient leurs troupeaux en deux groupes dont l'un, composé essentiellement de femelles en lactation, était localisé en zone périurbaine. Cette répartition du troupeau est une pratique de gestion utilisée par certains éleveurs pour diminuer les charges de travail, de complémentation, et pratiquer la vente de lait. Cette pratique n'était pas très développée dans la zone périurbaine d'Agadez dans la mesure où 24 p. 100 seulement des éleveurs enquêtés s'y adonnaient. La pratique de complémentation n'était pas non plus répandue en raison du mode d'élevage prédominant qui était le nomadisme. Les compléments alimentaires étaient constitués de graines de coton et de fanes de niébé.

\section{Gestion de la traite et lactation}

Plus de la moitié des éleveurs enquêtés (59 p. 100) vendaient du lait, soit à la laiterie Azla installée à Agadez, spécialisée dans la transformation de lait de dromadaire, soit au marché urbain traditionnel, soit à domicile chez des clients réguliers.

Chez les dromadaires, la descente de lait est conditionnée par la présence physique du chamelon à côté de sa mère. Chez les pasteurs touaregs enquêtés, la traite de la chamelle débutait plusieurs jours après la mise bas. L'administration du colostrum au chamelon était largement pratiquée dans la zone d'étude. En revanche, le nombre de jours entre la mise bas et la première traite était très 
variable. Il était fonction du nombre de chamelles suitées dans le troupeau et des besoins en lait de la famille. Parmi les éleveurs interrogés sur cette question, 63 p. 100 débutaient la traite trois semaines après la mise bas, 19 p. 100 deux semaines après et 18 p. 100 seulement quatre semaines après.

Le nombre de traites effectuées par jour joue un rôle important sur la quantité de lait que la chamelle peut produire. D'une manière générale 83 p. 100 des chameliers interrogés pratiquaient deux traites par jour (une le matin et une le soir au retour des chamelles), 10 p. 100 effectuaient trois traites par jour et sept éleveurs effectuaient une seule traite par jour. La traite était en général effectuée par deux personnes, mais elle peut être pratiquée par une seule personne à condition que l'éleveur soit expérimenté (figure 5). La traite était généralement faite par les femmes et les enfants, rarement par les hommes. La première tétée des trayons par le chamelon permettait de déclencher la descente du lait et d'accéder aux mamelles de la chamelle lors de la traite. La traite commençait quelques secondes (environ 10 à $15 \mathrm{~s}$ ) après la tétée du chamelon. En général la traite n'était jamais complète pour que le chamelon puisse disposer d'une certaine quantité de lait.

Ainsi la quantité de lait traite chez les chamelles dépendait non seulement de la capacité de production de la femelle, de la présence physique de son chamelon, mais également de l'expérience du trayeur. La traite était pratiquée au niveau de tous les élevages. Le taux de traite (nombre de chamelles traites sur le nombre total de chamelles suitées dans le troupeau) était en moyenne égal à 1 . Le récipient de traite le plus utilisé dans la zone 2 et 3 était l'akkbar (récipient en bois en forme de mortier destiné exclusivement à la traite). En zone 1, les éleveurs utilisaient plutôt des tasses en plastique ou métalliques. Les pasteurs chameliers lavaient systématiquement ces récipients avant la traite à l'eau. En revanche, très peu d'entre eux (33 p. 100) lavaient les trayons avant et après la traite.

Le lait trait était dans la majeure partie autoconsommé ; 41 p. 100 des éleveurs interrogés ne vendaient pas l'excédent (quantité obtenue après avoir enlevé la ration familiale) de leur production et, parmi eux, 11 p. 100 en faisaient don ; 25 p. 100 n'avaient généralement pas d'excèdent ; 3 p. 100 affirmaient verser l'excédent de leur production ; 2 p. 100 transformaient l'excédent de leur production en fromage. Ces excédents étaient en général enregistrés en période de production importante. Pour 57 p. 100 des chameliers, la période où ils enregistraient le plus d'excédents était pendant la

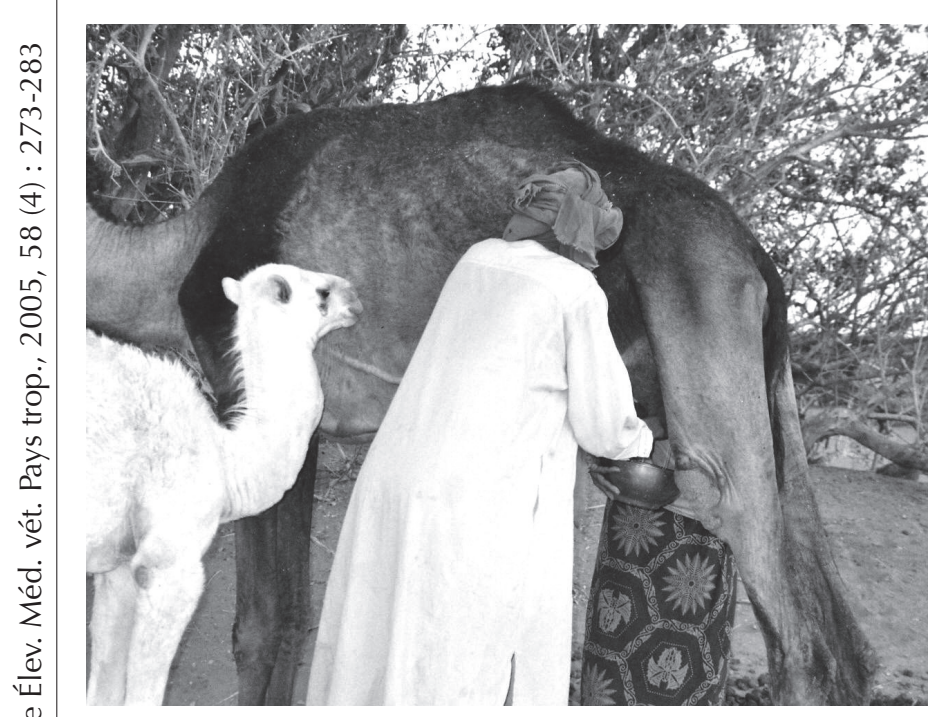

Figure 5 : la traite pratiquée par deux personnes nécessite la présence du chamelon. saison pluvieuse, alors que 38 p. 100 d'entre eux enregistraient le maximum de production en saison sèche froide. La vente du lait était du ressort de la femme en général.

\section{Gestion de la reproduction et sevrage}

La gestion de la reproduction était caractérisée par la présence d'un reproducteur eimalé (nom en tamasheq d'un mâle reproducteur) en permanence dans le troupeau de chamelles laitières. L'âge de la mise en reproduction pour les mâles était en moyenne de cinq ans alors qu'il était de trois ans et demi pour les femelles. Pour les éleveurs qui n'en possédaient pas, l'éleveur pouvait emprunter un mâle reproducteur qu'il introduisait dans son troupeau des femelles pour la saillie.

Au total, 69 p. 100 des éleveurs interrogés possédaient un mâle reproducteur dans leur troupeau, alors que 25 p. 100 n'en possédaient pas. Dans les troupeaux importants pouvaient se trouver deux mâles reproducteurs (6 p. 100), généralement l'un plus jeune que l'autre. En dehors des reproducteurs, les autres mâles arrivés à maturité sexuelle étaient en général castrés (affoda: nom en tamasheq des dromadaires mâles castrés ou non) et destinés au bât ou à la selle. Parmi les chameliers interrogés, 76 p. 100 possédaient un à cinq dromadaires adultes mâles castrés, 23 p. 100 n'en possédaient aucun, et 1 p. 100 en possédait plus de cinq. La fréquence d'avortements était relativement élevée dans cette zone. Des pourcentages de gestations avortées de 60 p. 100 ont été rapportés par certains chameliers. Mais seulement la moitié des éleveurs interrogés (50 p. 100) ont affirmé avoir enregistré des avortements à hauteur de 20 p. 100 des gestations. La mortalité des chamelons est également un aspect extrêmement important en élevage camelin. Les principales causes incriminées par les pasteurs étaient les diarrhées et les parasitoses externes (67 p. 100 des cas de mortalité).

La pratique de sevrage de chamelon était quasi inexistante dans la zone enquêtée. En effet, le chamelon était dans la plupart des cas sevré par sa mère, très souvent trois à quatre mois après la saillie fécondante. Seulement 7 p. 100 des éleveurs pratiquaient le sevrage volontaire des chamelons contre 93 p. 100 qui n'en pratiquaient pas du tout.

\section{Mobilité et occupation de l'espace}

La mobilité était la principale caractéristique des éleveurs de cette zone périurbaine d'Agadez (figure 6). Dans la zone 1, les éleveurs pratiquaient la complémentation durant pratiquement toutes les saisons. C'est cette complémentation qui stabilisait les animaux et limitait parfois leurs déplacements trop loin des parcs. Dans la zone 3 , où aucune complémentation n'était pratiquée, les éleveurs étaient en perpétuel déplacement. Dans cette zone, les dromadaires étaient en divagation et se déplaçaient sur de longues distances pour pâturer. Les éleveurs se déplaçaient en fonction de l'emplacement des points d'eau et des bons pâturages. Dans la zone 2, les éleveurs dans leur majorité se déplaçaient sur de faibles distances et ne le faisaient que chaque six mois (et dans de rares cas chaque deux à trois mois).

Certains éleveurs pratiquaient la transhumance en saison des pluies. Ce déplacement était effectué vers des zones lointaines (plus de 100 à $150 \mathrm{~km}$ vers l'ouest) jusqu'à In'Gall ou vers la zone pastorale du Tamesna. Cette migration des troupeaux durait en général deux à trois mois. Parmi les éleveurs enquêtés, 59 p. 100, dont la quasi-totalité des éleveurs de la zone 2, la pratiquaient. Cependant, beaucoup d'éleveurs ont affirmé y avoir eu recours en saisons difficiles. Pour certains éleveurs de la zone 1, elle concernait de façon irrégulière une partie du troupeau seulement, en général des femelles non suitées, et des mâles et des animaux en âge de mise en reproduction. Parmi les 28 chameliers interrogés dans cette zone, 


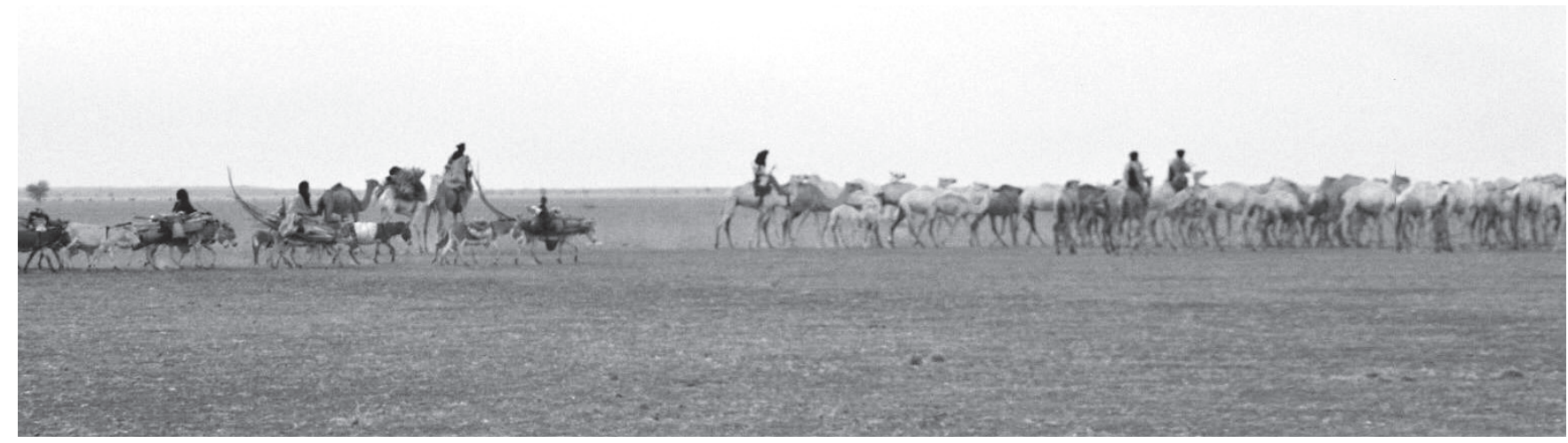

Figure 6 : la mobilité, une caractéristique importante de ce système.

19 p. 100 avaient adopté ce déplacement saisonnier d'une partie seulement du troupeau.

A travers cet ensemble de pratiques de gestion fonctionnelle des élevages, les auteurs ont essayé de réaliser une typologie. Les variables discriminantes obtenues, séparées en variables actives et supplémentaires à partir de différents thèmes et organisées en modalités avec les réponses pour chacune d'elles, ont été présentées dans le tableau II.

\section{DISCUSSION}

L'élevage camelin au Niger a bénéficié dans les années 1980 d'un certain intérêt des autorités publiques et des organisations non gouvernementales, dont les actions étaient orientées plutôt vers l'amélioration sanitaire, l'information et quelques interventions pilotes de développement (22). Cependant les mutations environnementales et socio-économiques ayant affecté les pays subsahariens ces dernières années remettent en cause très souvent un certain nombre d'acquis en termes de connaissance des systèmes d'élevages et de leurs performances (14). Dans tous les cas, les données bibliographiques concernant les systèmes d'élevages camelins au Niger et, à plus forte raison, les données sur leurs performances sont rares. Si elles existent, elles sont souvent fragmentaires, quelques fois incomplètes, pas assez précises pour constituer une base conceptuelle et cognitive solide (18). Les informations obtenues dans le cadre de cette étude ont permis de préciser les types d'élevages camelins qui gravitaient autour de la zone périurbaine d'Agadez, leur fonctionnement en termes de gestion de l'alimentation, de la traite et de la reproduction des troupeaux, et surtout d'apporter des informations sur un aspect très important de ce système, à savoir la mobilité.

La répartition des éleveurs chameliers se faisait selon plusieurs facteurs, principalement les ressources fourragères, les points d'eau, les zones d'attache, et secondairement l'exploitation et la vente du lait. Les zones 2 et 3 présentaient peu de ressources pastorales en raison du type de substrat. Le recouvrement végétal de ces deux zones était faible et hétérogène, et ne permettait pas de supporter du bétail sur une longue période. On observait alors une grande mobilité des éleveurs de ces zones, traduite par des mouvements itératifs pour ceux de la zone 3 et un mouvement cyclique et longitudinal pour ceux de la zone 2 .

En zone 3 les troupeaux étaient importants, ce qui imposait une recherche des grands espaces et assez de fourrages pour satisfaire leurs besoins, d'où un nomadisme quasi perpétuel. Les éleveurs y restaient en année de pâturage abondant mais, nomadisant entre les importants points d'eau pastoraux, ils migraient ensuite sur de grandes amplitudes au sud-ouest ou à l'ouest pendant les années difficiles.
En zone 2 la taille des troupeaux était faible ; cependant, en raison de l'afflux des troupeaux d'autres zones, les pasteurs étaient forcés de partir en transhumance saisonnière. Les points d'eau permanents étaient relativement peu nombreux, sauf dans la zone 1. L'exploitation du capital animal était centrée sur la vente des animaux, essentiellement les petits ruminants. La vente des dromadaires n'était pas fréquente chez ces pasteurs. Elle variait d'un à deux dromadaires par an selon la taille de la famille. Cette vente de dromadaire permettait au pasteur de se ravitailler en vivres. Les pasteurs se livraient aussi de plus en plus à la vente du lait de chamelle. Cette activité s'est accrue ces deux dernières années avec les crises socio-économiques et l'évolution progressive de la mentalité de ces pasteurs sous l'angle de la spéculation laitière qui jadis était comme prohibée, d'une part, et la forte croissance démographique avec une urbanisation marquée entraînant une augmentation de la demande en laits et produits laitiers au niveau des centres urbains, d'autre part.

Dans la zone 1 où il y avait une sédentarisation progressive, un nombre appréciable de pasteurs répartissaient leurs troupeaux en deux groupes : les femelles suitées pourvoyeuses de l'essentiel du lait autoconsommé et commercialisé, qui restaient au niveau des campements durant la lactation, et le reste du troupeau conduit par un jeune chamelier dans des zones lointaines pour exploiter les grands espaces pastoraux. La complémentation des animaux, en l'occurrence des chamelles laitières et des jeunes chamelons, était de plus en plus pratiquée, surtout par les éleveurs de la zone 1, avec des sous-produits agricoles obtenus sur les marchés d'Agadez.

Le déplacement constituait une composante essentielle de la gestion du troupeau. Tous les types de déplacements étaient pratiqués en allant du nomadisme à la conduite journalière des troupeaux aux pâturages. Selon Bernus (3), le nomadisme est une activité pastorale qui consiste en l'exploitation d'un espace aux ressources précaires, variables et dispersées dans des zones complémentaires. Le nomadisme implique la mobilité totale d'un groupe humain grâce à un habitat transportable (tente des Touaregs, yourtes des Mongols...) ou suffisamment sommaire pour être reconstruit à chaque déplacement. Pour Saidu (21), le nomadisme se distingue de la transhumance qui ne concerne que les bergers conduisant périodiquement des troupeaux sur des pâturages saisonniers à partir d'une implantation permanente. L'un des facteurs déterminants serait la taille du troupeau.

Cependant, on sait que la recherche des meilleures conditions d'élevages, en particulier les bons pâturages et les points d'eau accessibles, sont d'une importance capitale dans ce déterminisme $(15,16)$. En effet, les éleveurs dont les troupeaux ont un effectif réduit sont contraints de les garder auprès d'eux toute l'année, car 
ils dépendent beaucoup de ces animaux pour leur subsistance en lait ou pour le transport. En revanche, les éleveurs dont les troupeaux ont un effectif élevé envoient en transhumance un certain nombre de dromadaires, répartissant ainsi les charges en termes de suivi, d'entretien et de gestion des troupeaux. Ceci est observable surtout pour les troupeaux de taille moyenne et à effectifs importants. Les élevages camelins de l'ensemble de la zone se présentaient comme des élevages laitiers car ils comportaient plus de 53 p. 100 de femelles laitières.

\section{- CONCLUSION}

L'enquête typologique a porté sur 100 exploitations qui ne représentaient que 13 p. 100 des élevages installés dans le bassin laitier. Bien que ce pourcentage d'échantillonnage soit faible, ce travail a permis d'en savoir un peu plus sur les élevages de la zone périurbaine d'Agadez, en particulier sur la composition des troupeaux et leurs caractéristiques typologiques. Les résultats d'enquête obtenus ont montré l'existence de trois types d'élevages, différenciés essentiellement sur la base des pratiques d'alimentation des animaux et sur la mobilité des pasteurs. A cela s'est ajoutée une affinité zonale (d'origine socio-historique et écologique) de certains éleveurs.

L'analyse factorielle des correspondances multiples a montré de façon claire les corrélations entre les neuf variables actives utilisées dans cette analyse. Parmi elles, trois (ModElev, FreqDplcmt, OrDplcmt) étaient discriminantes et décrivaient parfaitement la situation des élevages et leurs relations. Les deux premiers groupes s'opposaient de fait par le mode d'élevage, l'un mobile et l'autre sédentaire.

Le troisième groupe d'éleveurs était un peu intermédiaire, raison pour laquelle il était moins homogène et comportait des variantes se rattachant à l'un ou à l'autre des groupes précités en termes de pratiques d'élevage. La construction d'une dualité (variables, individus) apportait également une parfaite corrélation en fonction de chaque type de pratique. La classification ascendante hiérarchique effectuée à partir des coordonnées factorielles a permis de classer tous ces individus en trois classes distinctes.

La première regroupait les éleveurs à gros effectifs (totaux et des dromadaires) de troupeaux, pratiquant le nomadisme (à l'intérieur et vers l'extérieur du bassin laitier) mais dont la zone de prédilection était la zone 3, vendant du lait, et ne pratiquant que rarement la transhumance. La deuxième était formée d'éleveurs semi-sédentaires, car ils désertaient dans leur majorité leur zone de prédilection (zone 2) en saison des pluies pour raison de transhumance, avec des effectifs de dromadaires assez faibles; ils ne vendaient généralement pas le lait produit. La troisième était celle des éleveurs dont les effectifs d'animaux dans les troupeaux étaient moyens ; ils pratiquaient la complémentation et ne se déplaçaient guère sur de longues distances. Leur mode d'élevage (sédentarisme) était motivé par la vente du lait facilitée par leur proximité de la ville d'Agadez.

Néanmoins, les déplacements étaient très importants puisque c'était grâce à eux que l'élevage persistait dans ces milieux arides. Ces résultats obtenus sur les types d'élevages de la zone périurbaine d'Agadez et leur mode de fonctionnement à travers l'étude des pratiques de gestion des élevages doivent permettre au développeur de prendre des décisions concrètes en matière d'aménagement du milieu mais également sur le plan du développement économique et social. En outre, d'autres études sont nécessaires pour comprendre la dynamique réelle des mouvements des éleveurs, la territorialité et l'organisation des éleveurs eu égard à la dynamique éco-climatique et socio-économique à laquelle la zone est soumise.

\section{BIBLIOGRAPHIE}

1. ALOU H., 1985. Contribution à l'étude de l'élevage camelin au Niger: situation actuelle, propositions d'amélioration, perspectives d'avenir. Thèse Doct. vét., Eismv, Dakar, Sénégal, 122 p.

2. BENZECRI J.P., 1984. Pratique d'analyse des données. Paris, France, Dunod, $456 \mathrm{p}$.

3. BERNUS E., 1986. Mobilité et flexibilité pastorale face à la sécheresse. Equipe sociétés nomades dans l'Etat. Nomadisme : mobilité et flexibilité ? Orstom, Bull. Liaison (8) : 137-144.

4. BONNET P., éd. sci., 1998. Actes colloque Dromadaires et chameaux, animaux laitiers, Nouakchott, Mauritanie, 24-26 oct. 1994. Montpellier, France, Cirad, $304 \mathrm{p}$

5. CHAIBOU M., FAYE B., 2003. Production laitière des chamelles Abzin élevées par les Touaregs nomades du Niger. In : Actes séminaire international sur le lait de chamelle, Niamey, Niger, 5-7 nov. 2003. Rome, Italie, FAO, p. 17-32.

6. DUTEURTRE G., MEYER C., éds sci., 2001. Actes de I'atelier international Marchés urbains et développement laitier en Afrique subsaharienne, Montpellier, France, 9-10 sept. 1998. Montpellier, France, Cirad, 233 p. (Coll. Colloques)

7. FAYE B., GOURCY E., BARNOUIN J., 1990. Méthodologie de description des systèmes alimentaires dans le cadre d'une enquête éco-pathologique en élevage bovin laitier. Epidémiol. Santé Anim., 17 : 117-134.

8. GIAZZI F., 1994. Sécheresse et occupation de l'espace dans le massif de l'Aïr. Sécheresse, 15 : 227-235.

9. LANDAIS E., LHOSTE P., 1987. Concepts et méthodes pour l'analyse du fonctionnement des systèmes d'élevage. In : Séminaire Economie et sociologies rurales, groupe méthodes et concepts, Montpellier, France, sept. 1989. Montpellier, France, Cirad, 30 p.

10. LEGAY J.M., 1986. Méthodes et modèles dans l'étude des systèmes complexes. Cah. Rech. Dev., $11: 1-6$.

11. LHOSTE P., 1984. Le diagnostic sur le système d'élevage Cirad. Cah. Rech. Dev., 3-4 : 84-88.

12. LHOSTE P., 1988. Les spécificités des systèmes d'élevage des régions chaudes justifient-elles des méthodes d'études propres? Etud. Rech. Sys. agraires Dév., 11 : 103-116.

13. MICHEL J.F., 1997. Contribution à l'étude des systèmes d'élevage camelin dans la province de Laâyoune au Maroc : enquête typologique. Thèse Doct. vét., Envt, université de Toulouse, France, 119 p.

14. MOULIN C.H., 1993. Le concept du fonctionnement de troupeau. Diversité des pratiques et variabilités des performances animales dans un système agropastoral sahélien. Etud. Rech. Sys. agraires Dév., 27 : 73-93.

15. MOULIN C., GIRARD N., DEDIEU B., 2001. L'apport de I'analyse fonctionnelle des systèmes d'alimentation. Fourrages, 167 : 337-363.

16. OSTY P.L., LANDAIS E., 1993. Fonctionnement des systèmes d'exploitation pastorale. In : Gaston A., Kernick M., Le Houérou H.N., éds, $4^{\mathrm{e}}$ Congrès international Terres de parcours, Montpellier, France, 2226 avr. 1991. Montpellier, France, Cirad, p. 1137-1146.

17. PERROT C., LANDAIS E., 1993. Exploitations agricoles; pourquoi poursuivre la recherche sur les méthodes typologiques; dossier: méthodes d'études en milieu paysan. Cah. Rech. Dev., 33 : 13-23.

18. PEYRE DE FABREGUES B., 1972. Modernisation de la zone pastorale du Niger. Maisons-Alfort, France, lemvt, 306 p. (Etude agrostologique $n^{\circ} 33$ )

19. PEYRE DE FABREGUES B., 1989. Le dromadaire dans son milieu naturel. Revue Elev. Méd. vét. Pays trop., 42 : 127-132.

20. ROGNON P., 1991. Les sécheresses du Sahel replacées dans l'évolution climatique des vingt derniers millénaires. Sécheresse, 2 : 199-210.

21. SAIDOU A., 1986. Contribution à l'étude d'un système pastoral sahélien : la transhumance au Niger: ses aspects, son incidence et les perspectives d'avenir. Thèse Doct. vét., université de Dakar, Sénégal, 120 p.

22. THEBAUD B., 1988. Elevage et développement au Niger (quel avenir pour les éleveurs du Sahel 2). Genève, Suisse, BIT, 147 p.

23. WALISER B., 1977. Systèmes et modèles, introduction critique à I'analyse des systèmes. Paris, France, Le Seuil, 250 p.

Reçu le 07.02.2005, accepté le 23.06.2006 


\section{Summary}

Chaibou M., Faye B. Herding Strategies of Camel Husbandry in Agadez Suburban Area in Niger. Typological Survey

Camel livestock relies on natural forage resources, which are subjected to irregular rainfalls, poorly distributed in time and space. Mobility is one of the strategies used by herders to utilize these resources. However, it has been a while since socioeconomic and climatic changes, and demographic growth and urbanization have changed the rules of herd management. Know-how of dairy farm management is essential for the development of a dairy processing unit in Agadez, whose main activity focuses on camel milk transformation. To help know the various herd management types in Agadez suburban area, 100 camel herders were surveyed. Analysis of the data obtained on herd management practices and on global management of camel herds helped distinguish three main herd types, differentiated on the bases of animal feed practices, herders' mobility, and a marked preference of some herders for this particular area, for socio-historical and ecological reasons. The first group of herders were sedentary; they owned an average size herd, used feed supplementation and sold camel milk. The second group mostly used transhumance during the rainy season; some herders supplemented their animals, but almost none of the herders sold camel milk. Herders in the third group owned large herds and therefore practiced nomadism regularly. Milk sale became a more common practice through the special contacts established by some of the producers of this group with the dairy processing unit. Herd size and composition, and forage and water resources were the main factors that determined herd movements.

Keywords: Camelus dromedarius - Livestock management Classification - Suburban area - Agadez - Niger.

\section{Resumen}

Chaibou M., Faye B. Funcionamiento de la cría camellar de la zona periurbana de Agadez en Níger: estudio tipológico

El ganado camellar depende de los recursos forrajeros de los pastaderos naturales, cuyo desarrollo está expuesto a la variabilidad e incertidumbre de las precipitaciones, muy mal repartidas en el tiempo y el espacio. Para explotar estos pastaderos abiertos, una de las estrategias adoptadas por los ganaderos es la movilidad. Sin embargo, desde hace algún tiempo, los cambios climáticos, socioeconómicos y las condiciones provocadas por una urbanización intensificada imponen cada vez más cambios en las reglas de gestión de las ganaderías. El desarrollo en Agadez de una minicentral lechera, cuya actividad principal se centra fundamentalmente en la transformación de la leche de camella, requiere un perfecto conocimiento del funcionamiento de las ganaderías lecheras que van a abastecer esta central. Se realizó un estudio durante dos meses, en cien ganaderías de camellos de la zona periurbana de Agadez, con el fin de conocer el funcionamiento de las ganaderías de esta zona. El análisis de los datos recogidos sobre las prácticas de manejo de rebaños y el funcionamiento global de las ganaderías de pastores camelleros permitió diferenciar tres grandes tipos de ganaderías, cuya diferencia reside fundamentalmente en las prácticas de alimentación de los animales y en la movilidad de los pastores. A esto se añade una afinidad zonal de algunos ganaderos, cuyas causas son sociohistóricas y ecológicas. El primer grupo de pastores tenía la peculiaridad de ser sedentario, de tener un rebaño de tamaño medio y de practicar el complemento de alimentación y la venta de leche. El segundo grupo de pastores practicaba mucho la trashumancia en temporada de lluvias, algunos daban complementos alimenticios a sus animales. Sin embargo, la comercialización de la leche de camella era prácticamente inexistente entre estos pastores. Los camelleros que componían el tercer grupo tenían grandes rebaños y efectuaban, por consiguiente, un nomadismo regular. Debido a los contactos particulares establecidos entre algunos productores de este grupo y la central lechera, la venta de leche cada vez se practicaba más. El tamaño, la composición de los rebaños y los recursos forrajeros e hidráulicos constituían los principales factores determinantes de los desplazamientos de los ganaderos.

Palabras clave: Camelus dromedarius - Manejo del ganado Clasificación - Zona periurbana - Agadez - Níger. 